\title{
Sémont Maneuver for Benign Paroxysmal Positional Vertigo Treatment: Moving in the Correct Plane Matters
}

\author{
*Isabel Gebhart, *Carina Götting, *Sara-Lynn Hool, *Miranda Morrison, *Athanasia Korda, \\ $*$ Marco Caversaccio, †Dominik Obrist, and $*$ Georgios Mantokoudis \\ * Department of Otorhinolaryngology, Head and Neck Surgery, Inselspital, University Hospital and University of Bern; and †ARTORG \\ Center for Biomedical Engineering Research, University of Bern, Bern, Switzerland
}

Hypothesis: We aimed to investigate the effect of the head excursion angle on the success of the Sémont Maneuver (SM).

Background: SM is performed with the head turned by 45 degrees toward the unaffected ear. In clinical routine, it is unlikely that physicians can turn the head to a position of exactly 45 degrees. Moreover, it is unclear how possible deviations from 45 degrees would affect the outcome with SM. Methods: We used an in vitro model (upscaled by $\times 5$ ) of a posterior semicircular canal with canalithiasis to study head excursion angles ( $0-75$ degrees) and minimum waiting times in SM. Additionally, we measured actual head excursion angles performed by trained physicians during SM on a healthy subject.

Results: Successful canalith repositioning to the utricle was possible at head excursion angles between 21 and 67 degrees. Waiting time increased from 16 to 30 seconds with increasing deviation from 45 degrees. Angles larger than 67 degrees or smaller than 21 degrees did not lead to successful repositioning even after a waiting period of 5 minutes. Physicians set head excursion angles of 50 degrees \pm SD 4.8 degrees while performing the SM.

Conclusion: Angular deviations up to \pm 20 degrees from the ideal SCC plane (45 degrees) still allows for successful SM. Although the tested physicians tended to underestimate the actual head excursion angle by 5 degrees (and more), the success of SM will not be affected provided that the waiting time is sufficiently long. Further, the results suggest that the Brandt-Daroff maneuver is a form of habituation training rather than a liberatory maneuver. Key Words: BPPVCanalolithiasis-Semicircular canal-Sémont liberatory maneuver-Vertigo.

Otol Neurotol 41:xxx-xxx, 2020.
The Sémont liberatory maneuver (SM) is an efficient treatment for Benign Paroxysmal Positional Vertigo (BPPV) of the posterior semicircular canal (1). However, the effects of the optimal movement plane on treatment success have not been investigated.

BPPV is the most common cause in patients with dizziness or vertigo with an incidence ranging from

Address correspondence and reprint requests to Georgios Mantokoudis, M.D., Head of Otology and Cochlear Implant Department, University Department of Otorhinolaryngology, Head and Neck Surgery, Inselspital Bern, 3010 Bern, Switzerland; E-mail: georgios.mantokoudis@insel.ch, Website: http://hno.insel.ch

D.O. and G.M. shared last authorship.

This study was supported by the Swiss National Science Foundation 320030_173081.

The authors disclose no conflicts of interest.

This is an open access article distributed under the terms of the Creative Commons Attribution-Non Commercial-No Derivatives License 4.0 (CCBY-NC-ND), where it is permissible to download and share the work provided it is properly cited. The work cannot be changed in any way or used commercially without permission from the journal.

DOI: $10.1097 /$ MAO.0000000000002992
10.7 to 64.0 cases per 100,000 population $(1,2)$ and the life-time prevalence is estimated at $2.4 \%(2,3)$. BPPV is also the most frequent cause of recurrent vertigo with a recurrence rate of $\sim 50 \%$ (3). Furthermore, a large proportion of acute dizzy patients in the Emergency Department suffer from BPPV $(4,5)$.

The widely accepted underlying mechanism of BPPV is the occurrence of free-floating otoconial debris from the utricle which disturbs the endolymph flow in semicircular canals (SCC) (6). The posterior SCC (pSCC) is affected in $80 \%$ of patients (7), probably due to its proximity to the utricle and its inferior anatomical position in relation to earth's gravity compared with the other SCCs. Otoconia might pass into the semicircular canal where they either move freely in the canal (canalolithiasis) or adhere to the cupula itself (cupulolithiasis) (8).

Repositioning maneuvers for BPPV treatment are clearing the semicircular canal of the floating debris and favoring migration to the utricle where otoconia are usually resorbed (7).

The first repositioning maneuver was described by Brandt and Daroff (9) in 1980 (Brandt-Daroff 
[BD]-Maneuver) for the treatment of an underlying cupulolithiasis (10). Semont et al. (11) introduced the SM in 1988, followed by Epley in 1992 (12). Both maneuvers aim to treat pSCC-BPPV and are currently considered gold standard treatments (7). The SM shows a variable recovery rate ranging from 40 (13) to $87 \%$ $(14,15)$. Repetition of the SM might further increase the recovery rate to 90.3 to $94 \% \%$ after four maneuvers (16).

$\mathrm{SM}$ is performed with the patient sitting in the upright position with the head turned by 45 degrees toward the unaffected ear and quickly leaned to the affected side. After a waiting time, the patient is then rapidly tilted to the unaffected side still with head turned by 45 degrees toward the unaffected ear (7). There are different recommendations on how to perform the SM with regards to waiting times (variations from 30 seconds to 5 minutes) $(7,11,17)$ and angular velocity during tilt to the opposite site (7). Our previous study based on an in vitro model of the SCC suggested a waiting time of 45 seconds and more (18). Contrary to current concepts (19), angular maneuver velocity did not have a major impact on the repositioning success rate, which needs to be confirmed in vivo. In addition, we found that movements beyond the earth horizontal (head extension angles of 20 degrees) were more effective. This maneuver with an extended movement range was also called the Sémont PLUS maneuver (20). The term "'head excursion angle," however, describes the position of the head relative to the torso. " Head tilting angle"' refers to the total movement angle from sitting to a lying position and vice versa.

In clinical routine, it is unlikely that physicians can turn the head to a position of exactly 45 degrees (head excursion angle) without the help of additional tools such as specialized positioning goggles (ICS Impulse, GN
Otometrics, Taastrup, Denmark; http://www.icsimpulse.$\mathrm{com} /$ ) or three-dimensional positioning chairs (TRV or Epley Omniax) (21). Moreover, it is unclear how possible deviations from the ideal SCC plane (45 degrees) would affect the successful repositioning of canaliths with SM and if there is a difference in waiting time. Self-treatments using the Semont or BD maneuvers proved to be less effective (22); however, it remains unclear whether these effects are due to inadequate positioning angles and waiting times.

We aimed to investigate the effect of the head excursion angle and waiting time on the success of SM using a SCC model and to compare these results to actual head excursion angles set by trained physicians during SM.

\section{METHODS}

\section{In Vitro Model for pSCC BPPV}

We used a scaled in vitro model of the pSCC (upscaled by $\times 5$ ) for our experiments (Fig. 1A). This in vitro model for pSCC with canalolithiasis was already described in detail by Obrist et al. $(18,23)$. It consists of a PVC tube (LabMarket, Ludwigshafen, Germany), with an inner diameter of $1.5 \mathrm{~mm}$ (mimicking the membranous duct), an artificial ampulla and utricle and viscous fluid modeling the endolymph (Glycerintricapprylat; Blaser Swisslube, Hasle-Rüegsau, Switzerland). Ball bearings (steel microspheres with a diameter of $250 \mu \mathrm{m}$, MPS Micro Precision Systems, Biel, Switzerland) served as a surrogate for otoconia.

The model was mounted on a lever-arm device (Fig. 1B) to mimic a patient pivoting about the pelvis to perform an SM. This in vitro model with a lever arm device was already used to study tilting angles and minimum waiting times in SM (18). The lever-arm device was $72 \mathrm{~cm}$ long (corresponding to a standard distance from the pelvis to the SCC) and could be freely rotated to the left and right with respect to the axis of the lever arm
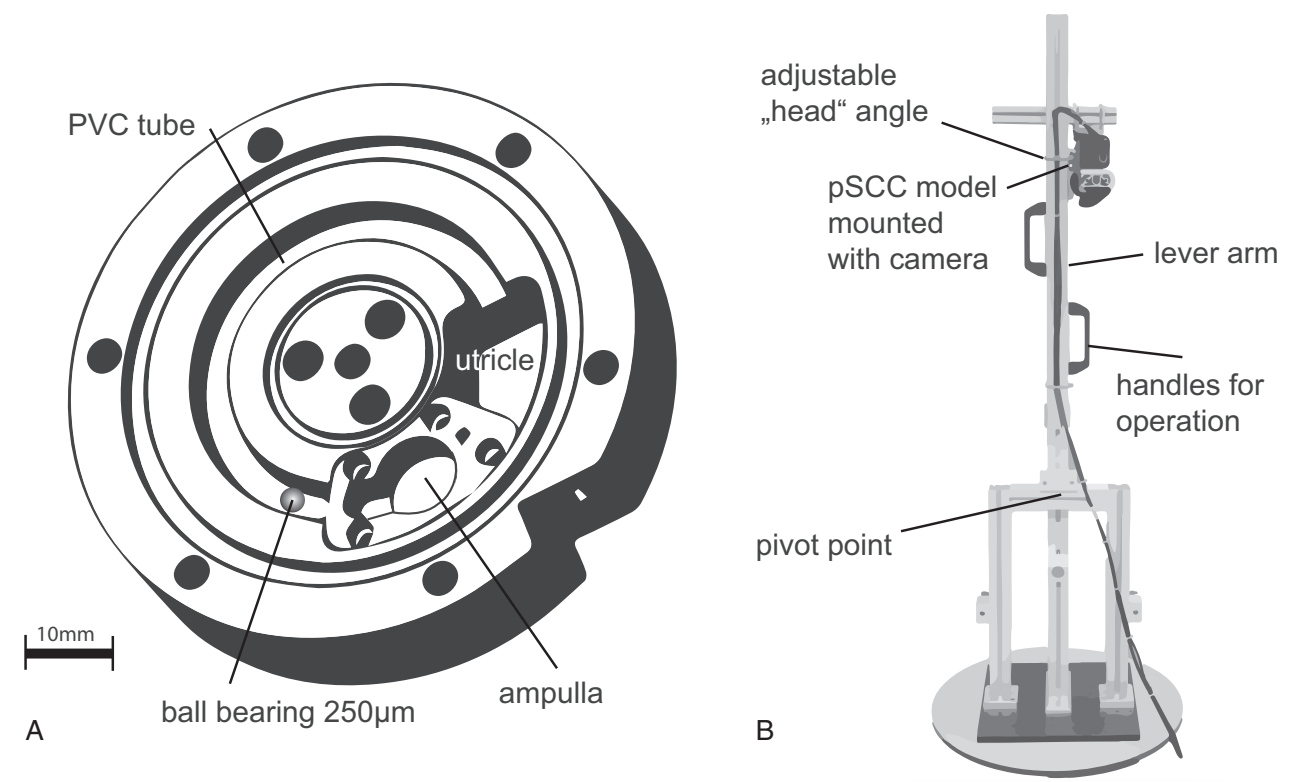

FIG. 1. Sketch from the pSCC model, sized 1:5 $(A)$ and the lever arm $(B)$ mimicking a sitting person performing the SM by pivoting about the pelvis. The orientation of the pSCC could be adjusted by using an angle scale with 1 degree units. pSCC indicates posterior semicircular canals; SM, Sémont Maneuver. Figure 1A adapted from Obrist et al., 2010. 
which corresponds to the head excursion angle during the SM. The mechanical stops on the left and right sides limited the range of tilting motions of the lever arm to 110 degrees $(90$ degrees tilting +20 degrees extension angle). This tilting angle was identified in the study of Obrist et al. (18) as an angle that yields good repositioning results for SM. A video camera (JAI RM-6740 GE, Stemmer Imaging, Pfäffikon, Switzerland) was fixed on the lever arm so that it moved with the SCC model to record videos.

\section{Experimental Protocol}

The experiments with the lever-arm were preformed with different waiting times and different head excursion angles for the SM. For simplicity, only one particle was used. At the beginning of each experiment, the SCC model was positioned such that the angle between the horizontal and the ampulla amounted to 32 degrees which corresponds to the SCC orientation in upright position. The particle had settled at the lowest position in the SCC which is 58 degrees away from the ampulla.

The optimal SM described in our previous study (18) consists of three steps, starting at a primary, sitting position and head turned to the left (45 degrees angle): 1 st step: lying to the right side with an tilting angle of 110 degrees $(90$ degrees +20 degrees extension angle below the earth horizontal). 2nd step: lying to the opposite, left side with an angle of 220 degrees (180 degrees $+2 \times 20$ degrees extension angle below horizontal) and 3rd step: sitting position ( 90 degrees +20 degrees). Peak head velocity of 135 degrees/s remained constant within tests (18) and velocity was monitored by a gyroscope and a stop watch.

We varied the head excursion angles from 0 degree (head straight) to 90 degrees to the left in 5 degrees steps. Critical angles were tested in 1 degree steps. The SM was considered successful if the particle settled in the utricle or unsuccessful if it settled in the ampulla or remained in the SCC. As a primary endpoint, we recorded the minimal waiting times for each tested head excursion angle from 0 to 90 degrees (after step 1 of the SM) for a successful SM.

For a given excursion angle, we measured threshold waiting times using an iterative measurement approach. We started with 30 seconds waiting time using decremental 5 second steps and at critical times 1 second steps until the SM was unsuccessful. Waiting time threshold for a successful SM was determined if three of five SM trials were successful. When the SM was unsuccessful at 30 seconds we extended the waiting time to maximum 5 minutes. Beyond 5 minutes, we considered the otoconia being irreversibly trapped in the SCC at the tested excursion angle.

\section{Measurement Head Excursions Angles by Trained Physicians}

We included nine trained ENT residents aged from 26 to 37 (mean $30 \pm \mathrm{SD} 3.1$ ) at tertiary referral hospital. They were instructed to choose correct 45 degrees head excursion angles on a healthy subject as if they were preforming SM. They repeated SM at least 10 times. Head excursion angle was recorded with a gyroscope mounted on a goggles frame and analyzed with MATLAB (MathWorks, Natick, MA).

\section{RESULTS}

\section{In Vitro Model for pSCC BPPV}

Figure 2 illustrates the results from the in vitro model. Successful canalith repositioning to the utricle was possible at head excursion angles between 21 and 67 degrees. The waiting time for the successful maneuvers increased with increasing deviation of the angles from 16 to 30 seconds. The angle of 45 degrees showed the

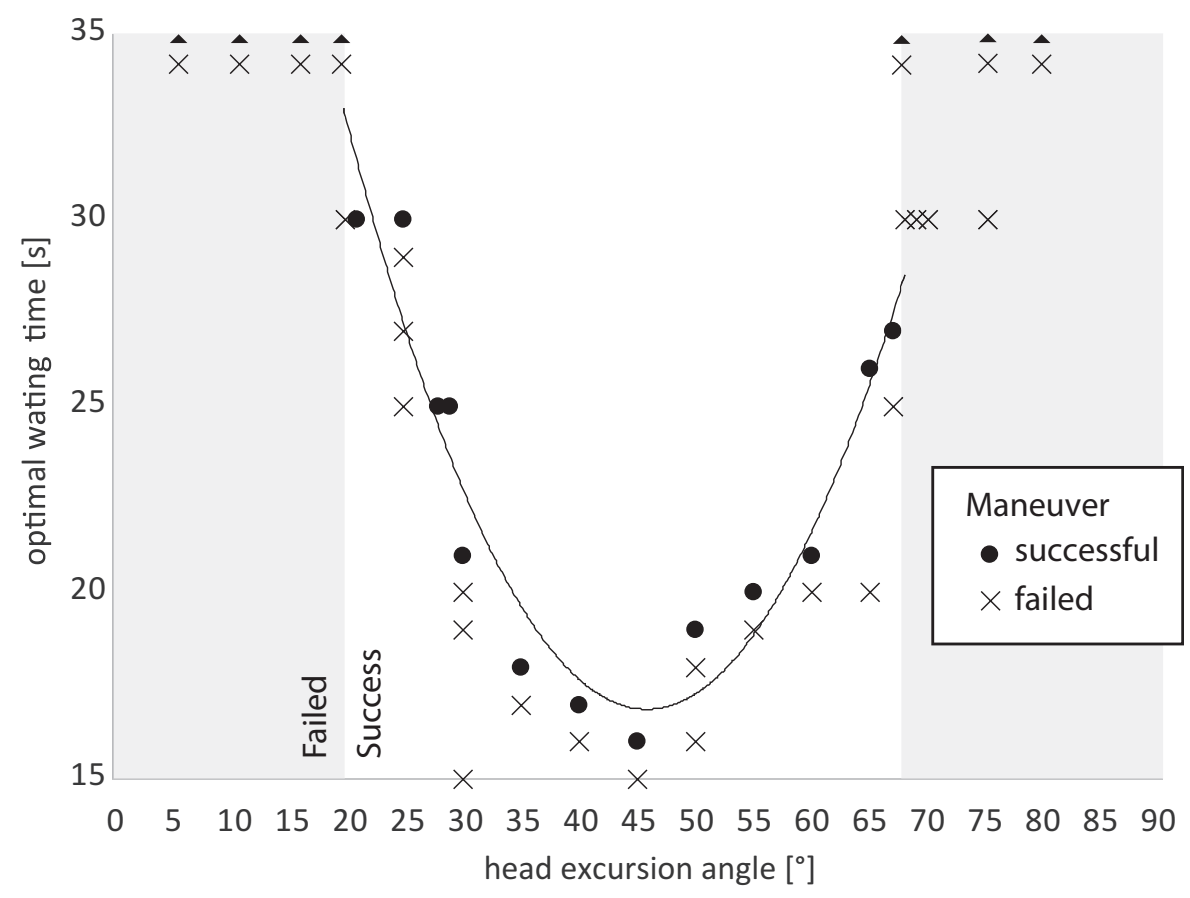

FIG. 2. Scatterplot showing waiting time thresholds in relation to head angle. Gray-shaded areas indicate head position angles with SM failure, even with a waiting time of 5 minutes. SM indicates Sémont Maneuver. 


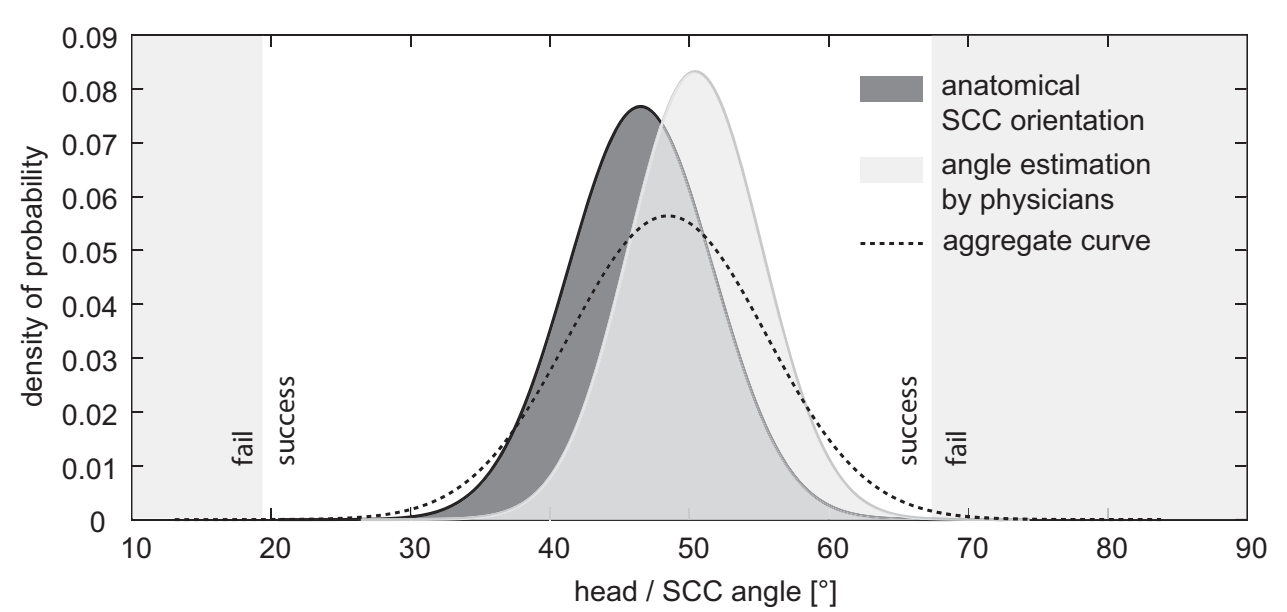

FIG. 3. Shows a density plot for the anatomical variations regarding $\mathrm{pSCC}$ plane and head angle estimations by physicians performing the $\mathrm{SM}$. The dotted line shows the aggregate curve with the overall probability of angle estimations during the SM. pSCC indicates posterior semicircular canals; SM, Sémont Maneuver.

smallest waiting time of 16 seconds. The lowest excursion angle of 21 degrees required a waiting time of 30 seconds and highest excursion angle 67 degrees required a minimal waiting time of 27 seconds.

Below the minimum waiting time of 16 seconds, the maneuver war not successful regardless of the chosen head angle. Head angles beyond 67 degrees or smaller than 21 degrees were never successful, even after a waiting time of 5 minutes. Thus, an offset of up to \pm 22 degrees from the optimal SCC plane was still resulting in a successful SM, provided that the waiting time was longer than 30 seconds and the head was tilted 20 degrees below the earth horizontal.

Waiting time for the SM increased rapidly with increasing deviation from the ideal angle 45 degrees (Fig. 2).

The maneuver was not successful because of three scenarios: 1) the artificial otoconium got stuck at the SCC wall and did not reach the lowest point of the SCC after the first step of the SM. After the second step of SM, the otoconium did not pass the apex of the SCC and fell back toward the initial position. 2) The otoconium reached the apex of SCC but got stuck at the SCC wall during the second step of the SM, and 3) the stuck otoconium did not move further toward the utricle after the 3rd step of the SM. We observed, however, one single and rare scenario, where the otoconium did not reach the utricle after the 2nd step but was successfully repositioned after the 3rd step.

\section{Measurement Head Excursions Angles by Trained Physicians}

Figure 3 illustrates the angle estimation by physicians and the anatomical SCC orientation. All physicians underestimated the head excursion angle while performing the SM. The mean head excursion angle of all physicians was 50 degrees \pm SD 4.8 degrees.

For comparison, Figure 3 compares the distribution of excursion angles set by the physicians to anatomical variations of the pSCC orientation which is described in anatomical studies to be positioned at 46.5 degrees \pm SD 5.2 degrees with respect to the azimuth Reid's plane (24). Figure 3 depicts also the overlapping of the normal distribution of anatomical variations of the SCC and the normal distribution of head angle estimations by physicians. The overall probability of repositioning failure due to wrong angle estimation and anatomical variability was $0.05 \%$ regarding the area under the curve for unsuccessful maneuvers (in Fig. 3).

\section{DISCUSSION}

The SCC plane during SM plays an important role in terms of positioning time and success rate: Waiting times increased rapidly with increasing angle offset from the plane of the pSCC. Large deviations from the pSCC plane and short waiting times prevented a successful SM. Imprecise angle estimations by physicians or anatomical variations were not the main reason for an unsuccessful maneuver.

\section{Sémont Maneuver}

The SM required a minimal waiting time of 16 seconds because the otoconium needed enough time to settle at the lowest point of the SCC before the second movement began. On the other hand, the otoconium needed also enough time to reach the utricle on the second and third step of the SM. According to Poiseuille's law, the flow velocity in the center of the SCC is greater than close to the SCC walls, which also has an effect on the velocity of otoconia. Otoconia positioned in the center of a cylindrical pipe might be in free fall due to gravity, opposed to otoconia migrating along the lateral wall (Fig. 4). In addition, we expect friction resistance for otoconia attached at the wall, slowing down velocity and thus, increasing the waiting time between each step of the maneuver. The velocity of the otoconia is also influenced by their size and shape. Furthermore, otoconia might never reach the apex of the SCC and thus, not being 

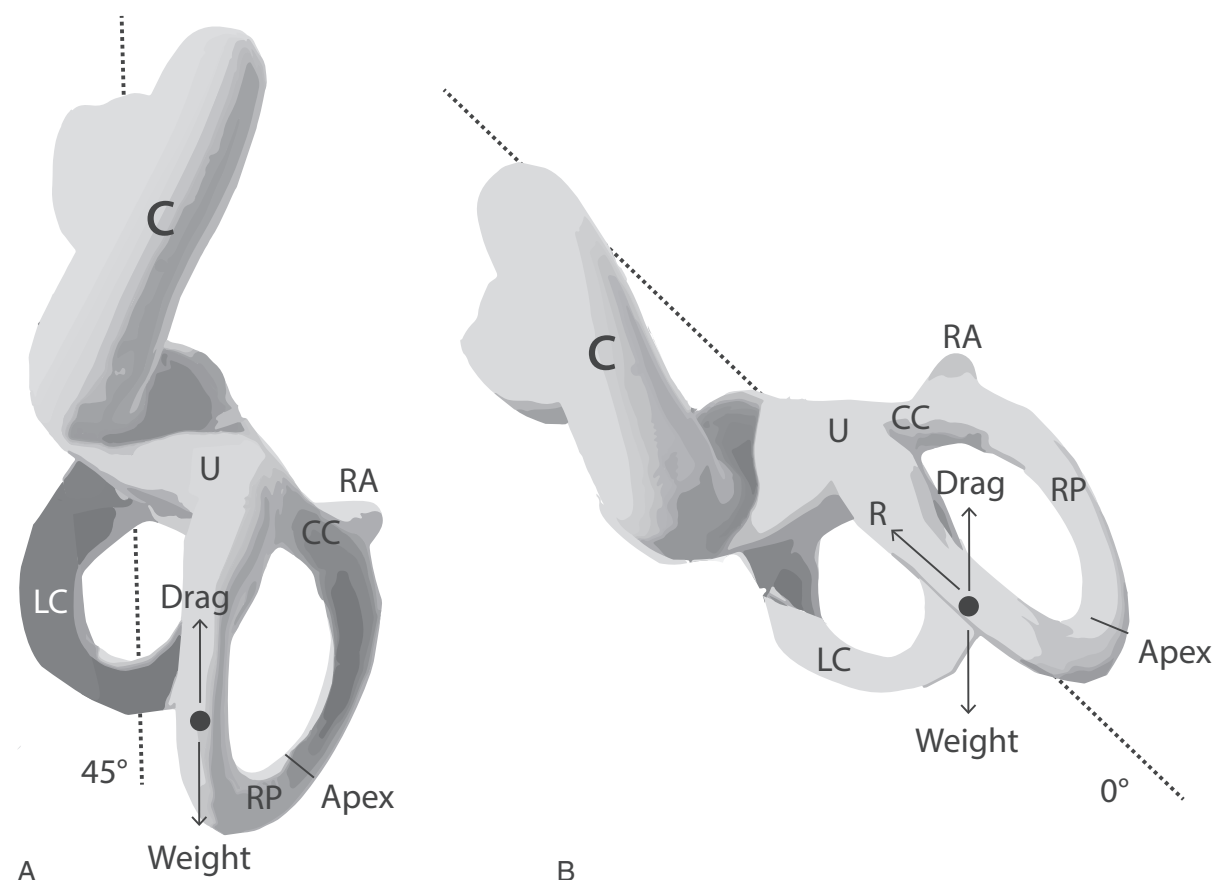

FIG. 4. Caudal view of the labyrinth and SCC orientation after the first step of SM using a 45 degrees angle $(A)$ or a 0 degree head excursion angle $(B)$, which corresponds to the first step of the Brandt-Daroff maneuver. AC indicates anterior canal; $C$, cochlea; CC, common crus; $L C$, lateral canal; PC, posterior canal; $\mathrm{R}$, resistance; $\mathrm{U}$, utricle. Please note the vertical orientation of the long arm of pSCC in $(A)$ and its oblique orientation in $(B)$.

pulled out by the second and third step of the SM. Finally, the anatomical geometry of the SCC and fluid dynamics of the endolymph have an impact on the velocity of the otoconia. Only in cases of extreme anatomical variations (pSCC angle at 46.5 degrees \pm SD 5.2 degrees) (24) failure of SM due to inadequate head excursion angle becomes possible.

Different recovery rates ranging from 70 to $90 \%$ have been observed for the SM $(16,25)$, due to different reasons: 1) Recovery rates are strongly dependent on the time interval between symptom onset and measurements. 2) There is a significant proportion of spontaneous recovery rate interfering with the reported results. 3) Repetition of the SM improves recovery rates. 4) There are different maneuver protocols and modifications reported in the literature. 5) There are different underlying causes such as trauma inducing a variation in severity of BPPV. 6) Multiple canals might have been affected. 7) There is an intersubject variability of SM parameters such as waiting times, excursion angles, velocities, and extension angles below the earth horizontal. 8) Outcome measures were based on different endpoints such as objective Dix-Hallpike results or subjective symptom recovery.

\section{Brandt-Daroff Maneuver}

BD maneuver corresponds to SM with a head excursion angle of 0 degree. For this configuration we were not able to successfully reposition otoconia. However, BD was considered equally effective as the Epley maneuver
(26) with a recovery rate of $64 \%$ in the first week and $88 \%$ after the second week; however, study numbers were low considering the high spontaneous recovery rate of more than $30 \%$ in BPPV (27). Another study showed that $\mathrm{BD}$ had a lower recovery rate of $50 \%$ compared with $73 \%$ with the SM (28). Larger RCTs confirmed the lower recovery rate of the BD maneuver being at 25 (29) and $24 \%$ (25), which is at the level of the spontaneous recovery rate. A Cochrane meta-analysis did not show any difference between the Epley and the SM maneuver but a superiority of these maneuvers compared with the BD (30). These findings are in line with our experimental study showing the inability of repositioning with such large head deviation angles. Therefore, observations of clinical improvement after BD maneuvers might rather be the result of spontaneous recovery or habituation effects.

\section{Strength and Limitations}

We tested only for SM and BD maneuver; however, our findings apply also partially to the Epley maneuver: The first part of the Epley maneuver is identical to the SM regarding SCC plane and head position. The Epley maneuver consists of several head positions, whereas the pSCC remains vertical in the first and the second last step of the Epley. Therefore, these two positions in the Epley maneuver are very critical regarding the vertically oriented canal plane and the waiting time needed. The Epley maneuver might therefore be even more susceptible to wrong head excursion estimations than the SM 
since the excursion angle between head and torso is changing multiple times.

All our findings are based on a model, using only a single otoconium for modeling BPPV and therapeutic maneuvers. Smaller otoconia, a larger number of otoconia, or even a formation of large clots forming plugs might behave differently during the maneuver. Our results are based on a model and might therefore not be generalizable. They need to be validated by clinical trials before being translated into clinics. However, our study still gives estimates derived from physical properties and it provides quantitative orientation for physicians regarding realistic and optimal waiting times and angles.

\section{Implications}

A waiting time of $>30$ seconds for each SM step turns out to be the most sensitive parameter influencing the success rate of the SM, provided that the patient was moved in the correct plane. This is in line with our previous in vitro experiment suggesting a waiting time of 45 seconds taking into account the size of the otoconia, the head velocity, and head position below earth horizontal (18). Self-administration of SM is less recommended due to the risk of inadequate performance $(2,22)$. Regarding the self-administration, we recommend patients to use a timer to respect the required waiting times. Professionals dealing with dizzy patients might provide a comprehensive guide (paper, video, or app) to instruct about adequate head and body positioning. New virtual reality goggles with build-in eye trackers might significantly improve the accuracy of the SM performed by patients or non-experts in the future. Specialists, however, might consider using three-dimensional chairs to apply standardized movements and extended positioning angles. Planned clinical trials need to take into account the timing and positioning parameters of the SM presented in this study.

\section{CONCLUSION}

Angular deviations up to \pm 22 degrees from the ideal SCC plane (45 degrees) still allow for successful SM. Although the tested physicians tended to underestimate the actual head excursion angle by 5 degrees (and more), the success of SM will not be affected provided that the waiting time is sufficiently long. Further, the results suggest that the Brandt Daroff maneuver (0 degree head excursion angle) is a form of habituation training rather than a liberatory maneuver.

\section{REFERENCES}

1. Cohen HS, Kimball KT. Effectiveness of treatments for benign paroxysmal positional vertigo of the posterior canal. Otol Neurotol 2005;26:1034-40.

2. von Brevern M, Radtke A, Lezius F, et al. Epidemiology of benign paroxysmal positional vertigo: A population based study. J Neurol Neurosurg Psychiatry 2007;78:710-5.

3. Neuhauser HK. The epidemiology of dizziness and vertigo. Handb Clin Neurol 2016;137:67-82.
4. Comolli L, Goeldlin M, Gaschen J, et al. [Dizziness and vertigo in a tertiary ENT emergency department]. HNO 2020;68:763-72.

5. Goeldlin M, Gaschen J, Kammer C, et al. Frequency, aetiology, and impact of vestibular symptoms in the emergency department: A neglected red flag. J Neurol 2019;266:3076-86.

6. Obrist D. Flow phenomena in the inner ear. Ann Rev Fluid Mech 2019;51:487-510.

7. Mandala M, Salerni L, Nuti D. Benign positional paroxysmal vertigo treatment: A practical update. Curr Treat Options Neurol 2019;21:66.

8. Choi S, Choi HR, Nahm H, et al. Utility of the bow and lean test in predicting subtype of benign paroxysmal positional vertigo. Laryngoscope 2018;128:2600-4.

9. Brandt T, Daroff RB. Physical therapy for benign paroxysmal positional vertigo. Arch Otolaryngol 1980;106:484-5.

10. Brandt T, Steddin S, Daroff RB. Therapy for benign paroxysmal positioning vertigo, revisited. Neurology 1994;44:796-800.

11. Semont A, Freyss G, Vitte E. Curing the BPPV with a liberatory maneuver. Adv Otorhinolaryngol 1988;42:290-3.

12. Epley JM. The canalith repositioning procedure: For treatment of benign paroxysmal positional vertigo. Otolaryngol Head Neck Surg 1992;107:399-404.

13. Lee JD, Shim DB, Park HJ, et al. A multicenter randomized doubleblind study: Comparison of the Epley, Semont, and sham maneuvers for the treatment of posterior canal benign paroxysmal positional vertigo. Audiol Neurootol 2014;19:336-41.

14. Mandalà M, Santoro GP, Asprella Libonati G, et al. Double-blind randomized trial on short-term efficacy of the Semont maneuver for the treatment of posterior canal benign paroxysmal positional vertigo. $J$ Neurol 2012;259:882-5.

15. Chen Y, Zhuang J, Zhang L, et al. Short-term efficacy of Semont maneuver for benign paroxysmal positional vertigo: A double-blind randomized trial. Otol Neurotol 2012;33:1127-30.

16. Levrat E, van Melle G, Monnier P, et al. Efficacy of the Semont maneuver in benign paroxysmal positional vertigo. Arch Otolaryngol Head Neck Surg 2003;129:629-33.

17. Fife TD, Iverson DJ, Lempert T, et al. Practice parameter: Therapies for benign paroxysmal positional vertigo (an evidence-based review): Report of the Quality Standards Subcommittee of the American Academy of Neurology. Neurology 2008;70:2067-74.

18. Obrist D, Nienhaus A, Zamaro E, et al. Determinants for a successful semont maneuver: An in vitro study with a semicircular canal model. Front Neurol 2016;7:150.

19. Faldon ME, Bronstein AM. Head accelerations during particle repositioning manoeuvres. Audiol Neurootol 2008;13:345-56.

20. Strupp M, Vinck A-S, Bayer O, et al. Benign paroxysmal positional vertigo: The "Sémont PLUS maneuver" is more effective than the Sémont maneuver-a prospective multinational randomized singleblinded trial (2338). Neurology 2020;94:2338.

21. Richard-Vitton T, Seidermann L, Fraget $\mathrm{P}$, et al. [Benign positional vertigo, an armchair for diagnosis and for treatment: Description and significance]. Rev Laryngol Otol Rhinol (Bord) 2005;126:249-51.

22. Radtke A, von Brevern M, Tiel-Wilck K, et al. Self-treatment of benign paroxysmal positional vertigo: Semont maneuver vs Epley procedure. Neurology 2004;63:150-2.

23. Obrist D, Hegemann S, Kronenberg D, et al. In vitro model of a semicircular canal: Design and validation of the model and its use for the study of canalithiasis. J Biomech 2010;43:1208-14.

24. Della Santina CC, Potyagaylo V, Migliaccio AA, et al. Orientation of human semicircular canals measured by three-dimensional multiplanar CT reconstruction. J Assoc Res Otolaryngol 2005;6:191-206.

25. Soto Varela A, Bartual Magro J, Santos Perez S, et al. Benign paroxysmal vertigo: A comparative prospective study of the efficacy of Brandt and Daroff exercises, Semont and Epley maneuver. Rev Laryngol Otol Rhinol (Bord) 2001;122:179-83.

26. Cetin YS, Ozmen OA, Demir UL, et al. Comparison of the effectiveness of Brandt-Daroff Vestibular training and Epley Canalith repositioning maneuver in benign Paroxysmal positional vertigo long term result: A randomized prospective clinical trial. Pakistan $J$ Med Sci 2018;34:558-63. 
27. Imai $\mathrm{T}$, Ito $\mathrm{M}$, Takeda $\mathrm{N}$, et al. Natural course of the remission of vertigo in patients with benign paroxysmal positional vertigo. Neurology 2005;64:920-1.

28. Gupta AK, Sharma KG, Sharma P. Effect of Epley, Semont Maneuvers and Brandt-Daroff Exercise on Quality of Life in Patients with Posterior Semicircular Canal Benign Paroxysmal Positional Vertigo (PSCBPPV). Indian J Otolaryngol Head Neck Surg 2019;71:99-103.
29. Amor-Dorado JC, Barreira-Fernandez MP, Aran-Gonzalez I, et al. Particle repositioning maneuver versus Brandt-Daroff exercise for treatment of unilateral idiopathic BPPV of the posterior semicircular canal: A randomized prospective clinical trial with short- and long-term outcome. Otol Neurotol 2012;33:1401-7.

30. Hilton M, Pinder D. The Epley (canalith repositioning) manoeuvre for benign paroxysmal positional vertigo. Cochrane Database Syst Rev 2014;CD003162. 